\title{
Efinaconazole in the treatment of onychomycosis
}

\author{
This article was published in the following Dove Press journal: \\ Infection and Drug Resistance \\ I June 2015 \\ Number of times this article has been viewed
}

\author{
Shari R Lipner \\ Richard K Scher \\ Department of Dermatology, Weill \\ Cornell Medical College, New York, \\ NY, USA
}

\begin{abstract}
Efinaconazole 10\% topical solution is a new triazole recently approved for the treatment of onychomycosis. It inhibits fungal lanosterol $14 \alpha$-demethylase in the ergosterol biosynthesis pathway, has potent antifungal activity against dermatophytes, as well as activity against Candida spp. and non-dermatophyte molds, and showed promising results in clinical trials. This review summarizes the mechanism of action, in vitro and in vivo data, clinical trials, safety, and quality-of-life data of efinaconazole as it applies to the treatment of onychomycosis.
\end{abstract}

Keywords: efinaconazole, Jublia, onychomycosis, fungal infection, nail infection

\section{Introduction}

Onychomycosis is a fungal infection of the nail unit caused by dermatophytes, yeasts, and non-dermatophyte molds. It is a common disease, with a prevalence of $10 \%-12 \%$ in the US. ${ }^{1,2}$ It may cause both physical and psychological problems, as some patients present with pain, difficulty wearing shoes, secondary infection, and problems performing everyday functions due to the resulting nail dystrophy or unacceptable cosmetic appearance. The treatment objective is to eradicate the fungus and produce a normal nail. The therapeutic innovations both in the 1990s and this past year have led to superior efficacy, more rapid treatment courses, and fewer drug-drug interactions. However, despite these improvements, approximately $20 \%-25 \%$ of patients will not respond to treatment, ${ }^{3}$ and relapse rates are high $(10 \%-53 \%){ }^{4}$

There are four approved classes of antifungal drugs for the treatment of onychomycosis: the allylamines, azoles, morpholines, and hydroxypyridinones. ${ }^{5}$ The allylamines (eg, terbinafine) inhibit squalene epoxidase, a step in the ergosterol biosynthesis pathway. ${ }^{6}$ Oral terbinafine ( $250 \mathrm{mg}$ daily) taken for 6 weeks for fingernails and 12 weeks for toenails is considered the current systemic treatment preference in onychomycosis therapy $^{7}$ with a complete cure rate of $38 \%$ in 12 -week studies, and mycological cure rate of $70 \%{ }^{8,9}$

The second class of drugs is the azoles, which inhibit lanosterol $14 \alpha$-demethylase, another step in the ergosterol biosynthesis pathway. ${ }^{5}$ Two members of this class that are widely used in treating onychomycosis are oral itraconazole ${ }^{10}$ and off-label oral fluconazole. ${ }^{11}$ The approved dose of oral itraconazole is $200 \mathrm{mg}$ daily for 3 months (or an alternative pulse regimen) with a reported complete cure rate of $14 \%$ and mycological cure rate of 38\%. ${ }^{10}$ Although fluconazole is not US Food and Drug Administration (FDA) approved for the treatment of onychomycosis in the US, it is used extensively in other countries and to some extent off-label in the US. In a study 
of 362 patients with fingernail onychomycosis treated with oral fluconazole, complete cure rates were $48 \%$ in patients who received $450 \mathrm{mg}$ weekly, $46 \%$ in those who received $300 \mathrm{mg}$ weekly, and $37 \%$ in those who received $150 \mathrm{mg}$ weekly for up to 9 months. ${ }^{11}$

Another class of antifungals is the morpholines including topical amorolfine, which is approved for use in Europe but not in North America. ${ }^{12}$ Amorolfine inhibits $\Delta 14$-reductase and $\Delta 7-\Delta 8$-isomerase in the ergosterol biosynthesis pathway, thus depleting ergosterol. ${ }^{13}$ In one randomized controlled study, the combination of amorolfine nail lacquer and oral terbinafine compared to oral terbinafine alone resulted in a higher clinical cure rate $(59.2 \%$ vs $46 \%)$; complete cure rate was not reported. ${ }^{12}$

Finally, the hydroxypyridinone class includes topical ciclopirox, which has a poorly understood mechanism of action but may involve iron chelation or oxidative damage. ${ }^{14,15}$ The FDA-approved ciclopirox 8\% nail lacquer in 1999 for the treatment of mild-to-moderate onychomycosis of fingernails and toenails without lunula involvement, due to Trichophyton rubrum in immunocompetent patients. Complete cure rates are $5.5 \%-8.5 \%$, and mycological cure rates are $29 \%-36 \%$ with monthly nail debridement and removal of residual lacquer. ${ }^{16}$ Until recently, ciclopirox had been the only FDA-approved option in the US for patients who had contraindications to oral antifungal agents, or preferred a topical treatment to avoid blood monitoring and potential for systemic side effects.

Based on the potential drug-drug interactions and systemic side effects of oral agents and the poor-efficacy and time-consuming treatment courses of topical drugs, it is clear that there is a need for alternative and novel therapies. There has been a greater emphasis on topical agents due to their more favorable side-effect profile and lower risk for drug-drug interactions. Efinaconazole, a member of the azole class, previously called IDP-108 and KP-103, has potent antifungal activity against dermatophytes comparable to that of terbinafine and amorolfine, higher activity against Candida spp. than itraconazole, and has been comparable to terbinafine and more effective than amorolfine, ciclopirox, and itraconazole against non-dermatophyte molds. ${ }^{17}$ Triazoles (such as fluconazole and itraconazole) had been used orally previously but not topically for the treatment of onychomycosis. Their appeal derives from their moderate antifungal activity but broader spectra than allylamines and morpholines. ${ }^{18}$ Efinaconazole is the first azole FDA approved in the USA to be used topically in the treatment of onychomycosis. This review summarizes the mechanism of action, in vitro and in vivo data, clinical trials, safety, and quality-of-life
(QoL) data of efinaconazole as it applies to the treatment of onychomycosis.

\section{Mechanism of action}

Fungal cell membranes are composed of the structural compound ergosterol, which maintains membrane fluidity, creates a permeability barrier, and is essential for fungal cell viability. It is believed that loss of ergosterol affects cell membrane integrity and function and inhibits fungal cell growth. ${ }^{19,20}$

Using an ergosterol biosynthesis assay, it was shown that efinaconazole inhibited ergosterol biosynthesis in both Trichophyton mentagrophytes and Candida albicans, and was more active than two comparator drugs, itraconazole and clotrimazole. T. mentagrophytes hyphal morphology exhibited changes that were dependent on the efinaconazole concentration (Table 1). The authors concluded that the mechanism of action of efinaconazole is through inhibition of the ergosterol pathway, likely via $14 \alpha$-demethylase, with secondary degenerative changes. ${ }^{21}$

\section{Chemistry and formulation}

Efinaconazole was synthesized as an azoleamine derivative by Kaken Pharmaceutical Co, and its chemical names are 1-piperidineethanol, and $\alpha$-(2,4-difluorophenyl)- $\beta$-methyl4-methylene- $\alpha-(1 H$-1,2,4-triazol-1-ylmethyl)-, $\alpha R, \beta R$-, or $(2 R, 3 R)-2-(2,4-d i f l u o r o p h e n y 1)-3-(4-m e t h y l e n e p i p e r i d i n-1-$ yl)-1-(1H-1,2,4-triazol-1-yl)butan-2-ol. ${ }^{18}$ Its molecular formula is $\mathrm{C}_{18} \mathrm{H}_{22} \mathrm{~F}_{2} \mathrm{~N}_{4} \mathrm{O}$ with a molecular weight of $348.39 .{ }^{22} \mathrm{It}$ was FDA-approved in the US for the topical treatment of toenail onychomycosis due to T. rubrum and T. mentagrophytes in June 2014, and its trade name is Jublia. The treatment course is once daily for 48 weeks, using a flow-through brush applicator with application to the affected toenail and its undersurface, nail folds, nail bed, and hyponychium. Two drops are needed for the great toenails, and one drop

Table I Hyphal morphology changes with efinaconazole

\begin{tabular}{|c|c|}
\hline $\begin{array}{l}\text { Efinaconazole concentration: } \\
0.001-0.01 \mu \mathrm{g} / \mathrm{mL}\end{array}$ & $\begin{array}{l}\text { Efinaconazole concentration: } \\
0.1-10 \mu \mathrm{g} / \mathrm{mL}\end{array}$ \\
\hline $\begin{array}{l}\text { Shortening of interseptal } \\
\text { distance }\end{array}$ & $\begin{array}{l}\text { Nonuniform widths and flattening } \\
\text { of hyphae }\end{array}$ \\
\hline Globular swelling & $\begin{array}{l}\text { Separation of plasma membrane } \\
\text { from the cell wall }\end{array}$ \\
\hline Thickening of the cell & $\begin{array}{l}\text { Accumulation of electron-dense } \\
\text { granules between the cell wall and } \\
\text { the plasma membrane } \\
\text { Discontinuity of the plasma membrane } \\
\text { Degeneration of organelles }\end{array}$ \\
\hline
\end{tabular}

Notes: Data from Tatsumi et al. ${ }^{21}$ 
is needed for other affected toenails. No debridement is necessary. It is formulated as a $10 \%$ solution, containing $100 \mathrm{mg}$ of efinaconazole per gram of a clear, colorless to pale yellow solution. Inactive ingredients are alcohol, anhydrous citric acid, butylated hydroxytoluene, C12-15 alkyl lactate, cyclomethicone, diisopropyl adipate, disodium edetate, and purified water. ${ }^{23}$ It has low surface tension, which aids in penetration and spreading, and is sparingly soluble in water. ${ }^{24}$ The drug accesses the site of infection by both transungual delivery ${ }^{25}$ and spreading through the subungual space. ${ }^{26}$ It is supplied in $4 \mathrm{~mL}$ or $8 \mathrm{~mL}$ bottles and should be stored at room temperature. While not published by manufacturer, judging from patient use, there are approximately 80 drops in a $4 \mathrm{~mL}$ bottle. Since there have been no studies of the drug in pregnant women, it is labeled pregnancy class $\mathrm{C}$, with a warning that it "should be used during pregnancy only if the potential benefit justifies the potential risk to the fetus". With repeated subcutaneous administration, efinaconazole was found in milk of nursing rats; however, it is unknown if efinaconazole is excreted into human milk. Therefore, the FDA states that caution should be exercised when efinaconazole is prescribed to women who are breastfeeding. ${ }^{23}$

\section{In vitro and in vivo studies}

In vitro studies showed that efinaconazole had potent activity against C. albicans, Cryptococcus neoformans, Aspergillus fumigatus, and T. mentagrophytes, similar to other synthesized azoleamine agents. ${ }^{18}$ Since the activities of many antifungal drugs are inhibited with keratin binding, ${ }^{27}$ efinaconazole's activity was tested against $T$. mentagrophytes in the presence of keratin. Efinaconazole demonstrated less deactivation than its comparators in the presence of keratin, which was attributed to its 4-methylenepiperidino group. In addition, it showed the highest efficacy compared to other drugs against $T$. mentagrophytes in a guinea pig model of tinea corporis and showed better penetration via both the transfollicular and transepidermal routes. ${ }^{18}$

Efinaconazole was subsequently shown to have excellent in vitro activity against $T$. rubrum and $T$. mentagrophytes, surpassing that of the reference drugs neticonazole and clotrimazole but less so than lanoconazole and butenafine. A similar effect was seen for the organisms, Trichophyton violaceum, Trichophyton ajelloi, Microsporum canis, Microsporum gypseum, and Epidermophyton floccosum. Efinaconazole was also more active against $C$. albicans, other Candida spp., and Malassezia furfur than the reference drugs. In addition, efinaconazole was the only agent whose activity against $T$. mentagrophytes was unaffected by the presence of serum or keratin. In vivo, topical efinaconazole achieved mycological cure in $80 \%$ of guinea pigs with experimental cutaneous candidiasis caused by $C$. albicans, but the reference drugs (clotrimazole, neticonazole, and lanoconazole) were ineffective in even decreasing the counts of fungi in the infected sites. In addition, in a guinea pig model of tinea pedis infected with $T$. mentagrophytes, 10 days of topical efinaconazole led to a dose-dependent therapeutic effect, which was almost maximal at a drug concentration of $1.0 \%$ and achieved negative culture results for all infected feet. This effect was considerably better than that of neticonazole and equal to that of lanoconazole and butenafine. The authors concluded that while the antidermatophytic activity of efinaconazole was similar to that of neticonazole and inferior to butenafine and lanoconazole, the drug might have superior efficacy in vivo due to its pharmacokinetics in infected skin tissues. $^{28}$

Based on these earlier findings, efinaconazole's efficacy was analyzed in guinea pig models of T. mentagrophytes interdigital tinea pedis and tinea corporis, as well as its ability to prevent relapses following treatment. The $1 \%$ efinaconazole solution resulted in a dose-dependent therapeutic effect in both infections, with all affected sites being culture negative. In the tinea pedis model, 30 days after the end of treatment (day 49 postinfection), relapse occurred in only 4/20 treated feet, while in the untreated control group, the infection continued without spontaneous healing. In the tinea corporis model, relapse occurred in only 3/10 animals treated with efinaconazole 9 days following treatment (day 21 postinfection), while the infection continued without spontaneous healing in the untreated group. In addition, when topical efinaconazole was applied 24-96 hours prior to T. mentagrophytes inoculation of skin in the tinea corporis model, the number of culture-positive animals 14 days postinfection was reduced. While this prophylactic effect was observed at all the recorded time points, the maximal effect was seen at 24 hours and 48 hours. Furthermore, when the binding capacity of efinaconazole was analyzed in an in vitro model, it was much lower $(60.3 \%)$ than either lanoconazole $(94.9 \%)$ or butenafine (99.4\%). Finally, efinaconazole was released more readily from keratin when washed with saline (84.7\% released) than lanoconazole (31.3\% released) and butenafine (5.2\% released). The investigators concluded that efinaconazole has the potential to achieve mycological cure in tinea pedis and tinea corporis infections and to prevent relapse. ${ }^{29}$

Investigators sought to overcome the problem with evaluating the treatment efficacy in animal models due 
to drug carryover in the treated tissues associated with conventional culture techniques. To do this, they developed a modified recovery culture method to remove antifungal drugs from treated tissue using stepwise dialysis with water, trypsin digestion, and phosphate-buffered saline rinses. Using this new method, in a guinea pig model of tinea pedis in which drug was applied topically for 10 days and then evaluated 5 days after the last application, they found that 20/20 lanoconazole-treated feet were culture negative with the conventional culture method, but only $3 / 20$ were culture negative with the newer method. In contrast, culturenegative rates were 19/20 and 17/20 in efinaconazole-treated feet with conventional and modified methods, respectively. Furthermore, using this updated method, they found that on posttreatment day $30,14 / 20$ of the infected feet treated with efinaconazole were culture negative, while the two reference drugs neticonazole and lanoconazole could not even diminish the fungal burden. ${ }^{30}$

In another study, it was shown that amorolfine and terbinafine were tightly bound to keratin and less active against dermatophytes in the presence of keratin, while efinaconazole is less tightly bound and exhibits better pharmacokinetics against fungi. It was also shown that efinaconazole was released more readily when bound to keratin than the other reference drugs. In addition, in a guinea pig model, while amorolfine and terbinafine reduced or eradicated the fungal burden in plantar skin tissues, these drugs were unsuccessful in reducing fungal burden in nails, likely via inactivation by keratin. On the other hand, efinaconazole was effective in both reducing the fungal burden in nails and curing infections in plantar skins, likely due to its lower affinity for keratin. ${ }^{31}$

In another report, the authors found that efinaconazole had a sevenfold higher free-drug concentration in the presence of keratin than both ciclopirox and amorolfine, and that this lower affinity for keratin was associated with faster nail penetration and fungicidal activity. They also found that efinaconazole inhibited growth of T. rubrum in an in vitro model of healthy human nails. ${ }^{32}$

In another recent study, the investigators characterized the in vitro antifungal activity of efinaconazole by analyzing its activity against recent clinical isolates recovered from onychomycosis patients (T. rubrum, T. mentagrophytes, and $C$. albicans isolates) and comparing that activity to that of terbinafine, ciclopirox, itraconazole, and amorolfine. They showed that efinaconazole had potent antifungal activity within a narrow concentration range (ie, $\leq 0.002-0.06 \mu \mathrm{g} / \mathrm{mL}$ ), with no geographical differences in susceptibility patterns between
North American (US and Canada) and Japanese isolates. ${ }^{17}$ It should be noted that the efinaconazole minimal inhibitory concentrations (MICs) in this study were approximately 13-63 times lower than previously reported in a study of Japanese T. rubrum and T. mentagrophytes clinical isolates. ${ }^{28}$ The authors attribute this difference to different culture techniques in the two studies. ${ }^{17}$ Using the $\mathrm{MIC}_{50}$ and $\mathrm{MIC}_{90}$ data, they showed that efinaconazole was at least as effective against $T$. rubrum and T. mentagrophytes (one- to fourfold) as amorolfine and terbinafine and higher (eight- to 64-fold) than ciclopirox and itraconazole. They also showed that efinaconazole was more potent in inhibiting C. albicans growth than terbinafine, ciclopirox, itraconazole, and amorolfine. They also studied efinaconazole's antifungal activity in 33 other fungal species and found that the drug was active against all species tested, with MIC ranges of $\leq 0.002-0.5 \mu \mathrm{g} / \mathrm{mL}$ for dermatophytes, $\leq 0.002-0.13 \mu \mathrm{g} / \mathrm{mL}$ for yeasts, and $0.0078-2 \mu \mathrm{g} / \mathrm{mL}$ for non-dermatophyte molds. On the whole, efinaconazole was more active against molds and yeasts than amorolfine, ciclopirox, itraconazole, and terbinafine, and its antifungal spectrum was broader than that of the existing drugs. ${ }^{17}$

Efinaconazole was tested for its ability to induce resistance in dermatophytes by continuous exposure of $T$. rubrum strains to the drug in vitro (12 passages) and in a guinea pig onychomycosis model ( 8 weeks) ${ }^{33}$ Resistance is an important feature to consider in treating patients, as topical treatments are typically used for a year and recurrences are common. ${ }^{34}$ Resistance may be due to a stress reaction in fungi with induction of drug efflux pumps. ${ }^{35}$ Interestingly, there was no suggestion of efinaconazole resistance in either the in vitro or in vivo experiments. ${ }^{33}$

\section{Pharmacokinetics}

Pharmacokinetic data were analyzed in healthy volunteers $(n=10)$ and patients with severe onychomycosis ( $\geq 80 \%$ surface area of both great toenails) $(n=20)$ in two single-center open-label studies (Phase I). Efinaconazole was applied to the healthy volunteers by staff on day 1 , and then on days 4-10, for a total of eight doses and applied to the onychomycosis patients daily for 28 days. ${ }^{36}$

The data showed that efinaconazole is absorbed slowly, lacks an elimination phase, ${ }^{36}$ and is metabolized through both oxidation and reduction, yielding an $\mathrm{H} 3$ metabolite. ${ }^{23}$ The concentration of efinaconazole and its metabolite was approximately 3.6-fold and 5.8-fold greater on day 10 than on day 1 in healthy volunteers and 6.8-fold and 30.5-fold greater on day 28 than day 1 in onychomycosis patients, indicating that the drug accumulates with successive applications. 
After five doses (day 8) in healthy volunteers and 14 doses (day 14) in onychomycosis patients, the drug and metabolite reached steady-state concentrations. The median half-life of drug and its metabolite at day 10 in healthy volunteers was 29.9 hours and 82.4 hours, respectively. In addition, the drug and metabolite were detected in plasma 2 weeks after the final dose in patients with onychomycosis. Taken together, the data suggest that efinaconazole and its metabolite have long elimination half-lives. Blood concentrations of efinaconazole and its metabolite were very low in both groups of patients, signifying that systemic exposure was negligible. Specifically, the concentrations of efinaconazole and its $\mathrm{H} 3$ metabolite were $1.47 \mathrm{ng} / \mathrm{mL}$ and $7.45 \mathrm{ng} / \mathrm{mL}$, respectively. ${ }^{36}$ This is in contrast to daily orally administered itraconazole (200 mg) and terbinafine (250 mg) with steady-state plasma level greater than $1,000 \mathrm{ng} / \mathrm{mL} .^{37,38}$ The investigators also noted that while efinaconazole is a potent inhibitor of several cytochrome P450 enzymes, its calculated $C_{\max } / k_{\mathrm{i}}$ is 0.007 and that of its metabolite is 0.0005 , both of which are well below the threshold for clinical drug-drug interactions. ${ }^{36}$ Protein-binding affinity is $95.8 \%-96.5 \%$, binding principally to albumin, $\alpha 1$-acid glycoprotein, and $\gamma$-globulin. ${ }^{23}$ The authors suggested that since efinaconazole is highly plasma protein bound, the potential for in vivo drug-drug interactions is remote. ${ }^{36}$

\section{Design and results from clinical trials \\ Phase II trial}

A multicenter, randomized, parallel-group, double-blind, vehicle-controlled study (NCT00777868) was conducted in Mexico in 135 patients with mild-to-moderate distal lateral subungual onychomycosis (DLSO) of the toenails. Primary endpoints were complete and mycological cure. Inclusion criteria and exclusion criteria are shown in Table 2. Patients who were taking medications that inhibit cytochrome P450 3A4 were not excluded.

Patients were randomized to one of four groups $(2: 2: 2: 1$ ratio), namely efinaconazole $10 \%$ topical solution with semiocclusion $(n=36)$, efinaconazole $10 \%$ topical solution $(n=39)$, efinaconazole $5 \%$ topical solution $(n=38)$, or vehicle $(n=22)$. The patients applied the medication once daily for 36 weeks, followed by a treatment-free follow-up period of 4 weeks. Demographics were similar between groups with mean age of 42.8 years and $54.1 \%$ female, and all were Hispanic/Latino. The mean area of the target toenail was $40.3 \%$, and the mean number of affected nontarget toenails (including great toenails and other affected toenails) was
Table 2 Inclusion and exclusion criteria for efinaconazole Phase II clinical trial

\begin{tabular}{|c|c|}
\hline Inclusion criteria & Exclusion criteria \\
\hline $\begin{array}{l}\text { DLSO affecting at least } \\
\text { one great toenail }\end{array}$ & Dermatophytoma (fungal abscess) \\
\hline Age $18-65$ years & Matrix (lunula) involvement \\
\hline $\begin{array}{l}\text { Clinical involvement } 20 \%-50 \% \\
\text { of the target toenail }\end{array}$ & $\begin{array}{l}\text { History of immunosuppression } \\
\text { and/or clinical signs indicative of } \\
\text { possible immunosuppression }\end{array}$ \\
\hline $\begin{array}{l}\text { Target toenail with uninfected } \\
\text { length (from the proximal nail } \\
\text { fold) } \geq 3 \mathrm{~mm}\end{array}$ & $\begin{array}{l}\text { Known human immunodeficiency } \\
\text { virus infection }\end{array}$ \\
\hline $\begin{array}{l}\text { Target toenail with } \\
\text { thickness } \leq 3 \mathrm{~mm}\end{array}$ & Uncontrolled diabetes mellitus \\
\hline Evidence of toenail growth & $\begin{array}{l}\text { Presence of toenail infection other } \\
\text { than dermatophytes and Candida }\end{array}$ \\
\hline $\begin{array}{l}\text { Positive potassium hydroxide } \\
\text { microscopy }\end{array}$ & $\begin{array}{l}\text { Severe moccasin-type tinea pedis } \\
\text { at the screening or baseline visits }\end{array}$ \\
\hline $\begin{array}{l}\text { Culture of a dermatophyte or } \\
\text { mixed dermatophyte/Candida } \\
\leq 42 \text { days before baseline visit }\end{array}$ & $\begin{array}{l}\text { Any disease/condition that might } \\
\text { have caused toenail abnormalities }\end{array}$ \\
\hline $\begin{array}{l}\text { Women of childbearing } \\
\text { potential had to be using } \\
\text { effective birth control }\end{array}$ & Previous target toenail surgery \\
\hline
\end{tabular}

Notes: Data from Tschen et al. ${ }^{39}$

Abbreviation: DLSO, distal lateral subungual onychomycosis.

4.9. Overall, $117(86.7 \%)$ of subjects completed the study. The most frequent reasons for study discontinuation were lost to follow-up ( $n=8,5.9 \%)$, subject request $(n=3,2.2 \%)$, and adverse events (AEs) $(n=3,2.2 \%)$. The data for complete and mycological cures at week 36 are shown in Table $3 .{ }^{39}$

Based on its greater efficacy in this Phase II trial, efinaconazole $10 \%$ solution (without semiocclusion) was chosen for treatment in the Phase III program. Another parameter that was analyzed was mean percent toenail involvement in the target toenail. At baseline, subjects had approximately $40 \%$ involvement. At week 36, percent involvement decreased to less than $19 \%$ in the efinaconazole-treated groups but was $35.7 \%$ in the vehicle group at week $36(P \leq 0.0215)$. Furthermore, the target toenail grew faster $(4.7 \mathrm{~mm}$ and $3.8 \mathrm{~mm}$, in the efinaconazole $10 \%$ and $5 \%$ groups, respectively) than in the vehicle group $(1.8 \mathrm{~mm})(P \leq 0.0196)$ and week 36. In terms of safety, adverse effects were generally mild (75\%-79.7\%) and similar between active drug and vehicle. Treatment-emergent adverse effects related to the study groups were blister, contact dermatitis, erythema, and ingrowing nail, none of which caused patients to discontinue the study. ${ }^{39}$

\section{Phase III trials}

Two identical, randomized, double-blind, vehicle-controlled Phase III studies (NCT01008033 and NCT01007708) were 
Table 3 Results from the four treatment arms in efinaconazole Phase II trial

\begin{tabular}{|c|c|c|c|c|}
\hline & $\begin{array}{l}\text { Efinaconazole } 10 \% \text { solution } \\
\text { with semiocclusion }\end{array}$ & $\begin{array}{l}\text { Efinaconazole } \\
10 \% \text { solution }\end{array}$ & $\begin{array}{l}\text { Efinaconazole } \\
5 \% \text { solution }\end{array}$ & Vehicle \\
\hline Complete cure (week 36) & $22.2 \%$ & $25.6 \%$ & $15.8 \%$ & $9.1 \%$ \\
\hline Mycological cure (week 36) & $83.3 \%$ & $87.2 \%$ & $86.8 \%$ & $\mathrm{~N} / \mathrm{A}$ \\
\hline $\begin{array}{l}\text { Mycologic cure and either an affected target toenail area } \\
\text { of } 0 \% \text { or }>3 \mathrm{~mm} \text { proximal nail growth from baseline } \\
\text { in the unaffected target toenail (week } 36 \text { ) }\end{array}$ & $61 \%$ & $64 \%$ & $55 \%$ & $23 \%$ \\
\hline$<20 \%$ of affected target toenail area (week 36 ) & $67 \%$ & $69 \%$ & N/A & $32 \%$ \\
\hline
\end{tabular}

Notes: Data from Tschen et al. ${ }^{39}$

Abbreviation: N/A, not available.

performed in patients with onychomycosis treated with efinaconazole $10 \%$ solution to assess the primary endpoint of complete cure rate at week 52 . The four secondary endpoints were mycological cure, complete or almost-complete cure (defined as less than or equal to $5 \%$ clinical involvement with mycological cure), treatment success (defined as $<10 \%$ clinical involvement of the target nail), and unaffected toenail growth. Other parameters investigated included change in the number of affected nontarget toenails, change in QoL, and target toenail growth. ${ }^{40}$

Patients qualified for the trial if they were 18-70 years old with clinical diagnosis of DLSO affecting $20 \%-50 \%$ of at least one great toenail, and had $3 \mathrm{~mm}$ of uninfected nail measured from the proximal nail fold, nail thickness $3 \mathrm{~mm}$ or less, and evidence of toenail growth. Potassium hydroxide and culture showing a dermatophyte or mixed infection had to have been positive within 42 days of starting treatment. Those patients with dermatophytomas or matrix (lunula) involvement were excluded. Immunosuppressed patients, as well as patients with uncontrolled diabetes mellitus, non-dermatophyte infection, severe moccasin tinea pedis, previous great toenail surgery, or other nail diseases, were also excluded. ${ }^{40}$

Patients were randomized to receive treatment with efinaconazole $10 \%$ solution or vehicle (3:1) self-applied to the nail plate and undersurface, lateral and proximal nail folds, and the hyponychium for 48 weeks. They were evaluated at weeks $0,12,24,36$, and 48 and then at 4 weeks posttreatment.

One thousand six hundred and fifty-five patients participated in the two studies with 118 sites in the US, Canada, and
Japan. Demographic data were similar for patients treated with efinaconazole and with vehicle. For studies 1 and 2, respectively, the mean ages were 52.3 years and 50.6 years, with the majority of patients being male $(74.4 \%$ and $80.4 \%$, respectively). Most patients were white (64.9\% and $87.8 \%$ ); however, in one study, there were a significant number of Asian patients (29\%), since there were 33 trial sites in Japan. Mean target toenail involvement was $36.7 \%$ and $36.3 \%$, and the mean number of nontarget nails involved was 2.8 in both studies. Total of $85.8 \%$ of patients completed the studies and of the patients who discontinued, reasons in order of frequency were patient request, lost to follow-up, adverse effects, protocol violation, other worsening condition, and pregnancy. ${ }^{40}$

For the primary endpoint, complete cure, $17.8 \%$ and $15.2 \%$ of patients on efinaconazole, in studies 1 and 2 , respectively, achieved the desired result at week 52, in contrast to patients on vehicle ( $3.3 \%$ and $5.5 \%, P<0.001)$. For the secondary endpoint of mycological cure, $55.2 \%$ and $53.4 \%$ of patients on efinaconazole achieved this result as compared to vehicle $(16.8 \%$ and $16.9 \%, P<0.001)$. Data for primary and secondary endpoints as well as other parameters are shown in Table 4.

The medication was well tolerated, and the AE profile was similar between patients treated with efinaconazole (66\% and 64.5\%) and with vehicle (61\% and 58.5\%). The most common AEs were nasopharyngitis, upper respiratory tract infection, and sinusitis (Table 5). Note that the numbers in Tables 4 and 5 vary due to patients who discontinued the trial. Very few patients discontinued the trial due to AEs (efinaconazole: $3.2 \%$ and $1.9 \%$, vehicle: $0.5 \%$ and $0 \%$ ), and

Table 4 Results from efinaconazole Phase III trials

\begin{tabular}{lllll}
\hline & $\begin{array}{l}\text { Efinaconazole I0\% solution } \\
(\mathbf{n = 6 5 6 )} \text { (study I) }\end{array}$ & $\begin{array}{l}\text { Vehicle (n=2I4) } \\
\text { (study I) }\end{array}$ & $\begin{array}{l}\text { Efinaconazole I0\% solution } \\
(\mathbf{n = 5 8 3}) \text { (study 2) }\end{array}$ & $\begin{array}{l}\text { Vehicle (n=202) } \\
(\text { study 2) }\end{array}$ \\
\hline Complete cure (primary endpoint) & $17.8 \%$ & $3.3 \%$ & $15.2 \%$ & $5.5 \%$ \\
Mycological cure & $55.2 \%$ & $16.8 \%$ & $53.4 \%$ & $16.9 \%$ \\
Complete or almost-complete cure & $26.4 \%$ & $7.0 \%$ & $23.4 \%$ & $7.5 \%$ \\
Treatment success & $35.7 \%$ & $11.7 \%$ & $31.0 \%$ & $11.9 \%$ \\
Unaffected toenail growth (mm) & 5.0 & 1.6 & 3.8 & 0.9 \\
\hline
\end{tabular}

Notes: Data from Elewski et al. ${ }^{40}$ 
Table 5 Most common adverse effects in efinaconazole trials

\begin{tabular}{lllll}
\hline & $\begin{array}{l}\text { Efinaconazole I0\% solution } \\
(\mathbf{n = 6 5 3 )} \text { (study I) }\end{array}$ & $\begin{array}{l}\text { Vehicle (n=2 I3) } \\
\text { (study I) }\end{array}$ & $\begin{array}{l}\text { Efinaconazole I0\% solution } \\
(\mathbf{n = 5 7 4 )} \text { (study 2) }\end{array}$ & $\begin{array}{l}\text { Vehicle (n=200) } \\
\text { (study 2) }\end{array}$ \\
\hline Nasopharyngitis & $78(11.9 \%)$ & $25(I I .5 \%)$ & $63(I 1.0 \%)$ & $15(7.5 \%)$ \\
Upper respiratory tract infection & $38(5.8 \%)$ & $13(6.1 \%)$ & $35(6.1 \%)$ & $11(5.5 \%)$ \\
Sinusitis & $30(4.6 \%)$ & $4(1.9 \%)$ & $17(3.0 \%)$ & $5(2.5 \%)$ \\
Eczema & $22(3.4 \%)$ & $7(3.3 \%)$ & - & - \\
Headache & $15(2.3 \%)$ & $5(2.3 \%)$ & $25(4.4 \%)$ & $7(3.5 \%)$ \\
\hline
\end{tabular}

Notes: Data from Elewski et al. ${ }^{40}$

the most common reason was application-site dermatitis and vesicles. $^{40}$

\section{Other safety data}

To address short- and long-term tolerability of efinaconazole, 239 healthy volunteers were enrolled in a study evaluating the likelihood of the drug to induce delayed contact skin sensitization and its skin irritation potential. For the contact sensitization study, there were induction, challenge, and rechallenge (if required) phases for both the drug and the vehicle. In $99.5 \%$ (206/207) of patients tested with drug and $99.0 \%(205 / 207)$ with vehicle, there was no evidence of contact sensitization. In the three patients who were rechallenged, the one patient who received efinaconazole did not have contact sensitization with occlusive, semiocclusive, or open applications. However, in the two patients who received vehicle upon rechallenge, there was likely an allergic reaction. Thirty-seven patients underwent a 21-day cumulative irritation study, receiving applications of efinaconazole $1 \%, 5 \%$, and $10 \%$ solutions as well as the positive control $0.2 \%$ sodium lauryl sulfate and the negative control deionized water. The calculated mean cumulative irritancy indices (based on erythema scores) were $1.12,1.26$, and 1.18 for the efinaconazole solutions, respectively, 1.04 for the vehicle, and 2.77 and 0.30 for the positive and negative controls, respectively. The authors concluded from their data that efinaconazole $10 \%$ solution did not cause contact sensitization and induces only minimal skin irritation. ${ }^{41}$

Safety data were also obtained from animal models. Efinaconazole solution and vehicle were administered dermally to mice (13 weeks), rats (6 months), and minipigs (9 months). Transient erythema, hyperkeratosis, and mild microscopic skin inflammation were seen. There was also minimal-to-moderate vacuolated changes in the rat dermal study but no systemic toxicity seen in mice and minipigs. In addition, the drug was not carcinogenic nor genotoxic in a 2-year mouse dermal study. Based on this data, the authors concluded that efinaconazole demonstrated low-to-moderate toxicity, similar to other azole antifungals, and a high safety margin for topical treatment of onychomycosis. ${ }^{42}$

The reproductive and developmental toxicity of efinaconazole was analyzed in rat models of fertility and early embryonic development, in rat and rabbit models of embryofetal development, and in rat models of peri-/postnatal development. Results showed that efinaconazole was similar to other approved azoles with regard to F1 mortality, placental effects, and estrous cycle prolongation. However, it has a more desirable safety profile than other azole antifungals, in that it is not teratogenic. In fact, efinaconazole did not affect embryo-fetal development in rabbits even when there was evidence of maternal toxicity. Furthermore, when maximal systemic exposures in trial subjects with onychomycosis treated topically with efinaconazole $10 \%$ solution were replicated in rats, no toxicity was observed. Developmental and reproductive toxicity was noted only at high ( $>100$-fold) multiples of human systemic exposure. ${ }^{43}$

\section{Studies in patients with diabetes}

Onychomycosis affects approximately one-third of patients with diabetes and may increase the severity of foot disorders such as nonhealing ulcers and secondary infections. ${ }^{44}$ Therefore, using data from the Phase III trials, a post hoc analysis was performed on 112 patients with diabetes, aged 29-70 years, randomized to daily application of efinaconazole $10 \%$ topical solution or vehicle. For the primary endpoint of complete cure, $13.0 \%$ of diabetics treated with efinaconazole achieved this result at week 52 compared with $3.7 \%$ diabetics treated with vehicle $(P<0.001)$, with no significant difference in efficacy between the diabetic and nondiabetic populations. For the secondary endpoint of mycological cure, $56.5 \%$ of diabetic patients achieved this result with active drug compared with $14.8 \%$ of diabetics treated with vehicle $(P=0.016)$, with no significant distinction between the efficacy of efinaconazole in the diabetic and nondiabetic populations. In addition, complete or almostcomplete cure rate with efinaconazole was $24.6 \%$ (vehicle, $7.4 \%$ ), and $40.8 \%$ of efinaconazole-treated patients had 
treatment success at week 52 vs vehicle (18.5\%). Finally, AEs with efinaconazole were similar to vehicle (64.2\% vs $66.7 \%$ ) and generally mild to moderate, similar to those seen in the total study population. ${ }^{45}$

\section{Studies on coexisting tinea pedis with onychomycosis}

Tinea pedis may occur in up to $50 \%$ of patients with onychomycosis ${ }^{46}$ and is considered a risk factor for the development of onychomycosis. ${ }^{47}$ Using data from the duplicate Phase III trials, a post hoc analysis was performed to analyze the efficacy of efinaconazole $10 \%$ topical solution in onychomycosis patients who also had coexisting tinea pedis. It was found that $21.3 \%(352 / 1,655)$ of onychomycosis study patients reported interdigital tinea pedis at baseline, ${ }^{48}$ which is consistent with previous reports. ${ }^{49}$ Although the studies were not designed to investigate tinea pedis treatment, investigator-approved topical antifungals were allowed. Two hundred and fifteen patients $(61.1 \%)$ with onychomycosis also had their coexisting tinea pedis treated in addition to being treated with efinaconazole. In patients with coexisting tinea pedis, who were treated concurrently for tinea pedis, complete cure rates with efinaconazole were $29.4 \%$ ( $P=0.003$ vs vehicle), and mycological cure rates were $56.2 \%(P \leq 0.001)$. On the other hand, when tinea pedis was not treated, complete cure rates were $16.1 \%(P=0.045$ vs vehicle $)$, and mycological cure rates were $45.2 \%(P=0.007) .{ }^{48}$ These data suggest that greater efficacy in onychomycosis treatment may be achieved with concurrent treatment of tinea pedis.

\section{Studies on patients with early and long-standing onychomycosis}

Using data from the duplicate Phase III trials, a post hoc analysis was performed to analyze the efficacy of efinaconazole $10 \%$ topical solution in onychomycosis patients with early and long-standing disease. At week 52, 42.6\% of patients with a baseline disease duration of $<1$ year had a complete cure with efinaconazole compared to $16.7 \%$ on vehicle; $17.1 \%$ of patients with a baseline disease duration of 1-5 years had a complete cure with efinaconazole $(P<0.001$ vs vehicle), and $16.2 \%$ of patients with baseline disease duration of $>5$ years had a complete cure $(P<0.001$ vs vehicle). In addition, $28.3 \%$ of patients with no other toenails affected at baseline had a complete cure with efinaconazole compared to $23.7 \%$ on vehicle $(P<0.001)$, and $18.6 \%$ and $16.5 \%$ of patients with $1-2$ or more than two nontarget toenails affected, respectively, at baseline had a complete cure with efinaconazole compared with vehicle (both $P<0.001$ ). Furthermore, in patients with no affected nontarget toenails at baseline, $8 \%$ of those treated with efinaconazole had 1-2 affected nontarget toenails at week 52 compared to $12 \%$ of patients treated with vehicle, and spread of onychomycosis to other toenails was greater in the vehicle group regardless of the baseline affected nontarget toenails. These results suggest that efinaconazole is more efficacious than vehicle irrespective of disease duration or number of nails affected and that treatment with efinaconazole may halt the spread of onychomycosis to other nails. ${ }^{50}$

\section{Studies using nail polish}

Since treatment of onychomycosis with efinaconazole may be for nearly a year, compliance and adherence to the treatment protocol are important for efficacy. Patients may want to camouflage their nails with nail polish during therapy. Therefore, a study was performed using normal human cadaver thumbnails polished with two coats of three different brands of redcolored nail polish and a control set of uncoated nails with treatment with one application of $\left({ }^{14} \mathrm{C}\right)$-efinaconazole $5 \%$ solution. The authors found that penetration of efinaconazole was not affected by nail polish. It should be noted that these cadaveric nails were non-diseased, treatment efficacy with nail polish use was not evaluated, ${ }^{51}$ and that the concentration of efinaconazole was different than that used in the Phase III studies ( $5 \%$ here vs $10 \%$ in Phase III). ${ }^{52}$

\section{QoL studies}

Onychomycosis may have substantial bearing on physical and psychological well-being, by disrupting daily life, negatively impacting QoL, and causing pain and difficulty with ambulation. ${ }^{53}$ Patients with onychomycosis have reported embarrassment, low self-esteem, and problems with social interaction due to their disease. ${ }^{54}$ Subjects who were part of the Phase III studies with efinaconazole were also assessed for a change in QoL with treatment using a self-administered OnyCOE-t ${ }^{\mathrm{TM}}$ questionnaire at baseline and at weeks 24 and 52 . The authors found that efinaconazole was significantly more effective than vehicle in improving QoL. In addition, there was a significant difference in the treatment satisfaction scores between those subjects who showed clinical improvement and those who did not improve. ${ }^{55}$

\section{Approval}

Efinaconazole 10\% solution (Valeant Pharmaceuticals International) was approved by Health Canada for the treatment of mild-to-moderate onychomycosis in October 2013. ${ }^{56}$ A regulatory application for approval of efinaconazole for 
the treatment of onychomycosis was submitted in Japan in 2012, and Kaken Pharmaceutical Co, Ltd, received approval in July 2014. ${ }^{57}$ Valeant Pharmaceuticals filed a new drug application seeking approval of efinaconazole in the US for the treatment of onychomycosis in July 2012, and it was FDA-approved in June 2014. There was a delay in approval due to questions regarding the closure of the container, but no efficacy or safety concerns were raised. ${ }^{58}$

\section{Conclusion}

Efinaconazole $10 \%$ topical solution is a new and promising treatment for mild-to-moderate DLSO onychomycosis. It has potent antifungal activity against dermatophytes, as well as activity against Candida spp. non-dermatophyte molds. It is appropriate for onychomycosis therapy in a broad range of patients due to its favorable side-effect profile, low rate of treatment-related AEs, and negligible risk for drug-drug interactions, and as there is no need for blood monitoring.

\section{Disclosure}

Dr Lipner has no conflicts of interest to disclose. Dr Scher is a consultant, investigator, and speaker for multiple companies including consultant and speaker for Valeant. The authors report no other conflicts of interest in this work.

\section{References}

1. Ghannoum MA, Hajjeh RA, Scher R, et al. A large-scale North American study of fungal isolates from nails: the frequency of onychomycosis, fungal distribution, and antifungal susceptibility patterns. J Am Acad Dermatol. 2000;43(4):641-648.

2. Scher RK, Rich P, Pariser D, Elewski B. The epidemiology, etiology, and pathophysiology of onychomycosis. Semin Cutan Med Surg. 2013;32(2 suppl 1):S2-S4.

3. Scher RK, Baran R. Onychomycosis in clinical practice: factors contributing to recurrence. Br J Dermatol. 2003;149(suppl 65):5-9.

4. Piraccini BM, Sisti A, Tosti A. Long-term follow-up of toenail onychomycosis caused by dermatophytes after successful treatment with systemic antifungal agents. J Am Acad Dermatol. 2010;62(3):411-414.

5. Welsh O, Vera-Cabrera L, Welsh E. Onychomycosis. Clin Dermatol. 2010;28(2):151-159.

6. Gupta AK, Sauder DN, Shear NH. Antifungal agents: an overview. Part II. J Am Acad Dermatol. 1994;30(6):911-933. [quiz 34-36].

7. Gupta AK, Paquet M, Simpson F, Tavakkol A. Terbinafine in the treatment of dermatophyte toenail onychomycosis: a meta-analysis of efficacy for continuous and intermittent regimens. J Eur Acad Dermatol Venereol. 2013;27(3):267-272.

8. Drake LA, Shear NH, Arlette JP, et al. Oral terbinafine in the treatment of toenail onychomycosis: North American multicenter trial. J Am Acad Dermatol. 1997;37(5 pt 1):740-745.

9. Evans EG, Sigurgeirsson B. Double blind, randomised study of continuous terbinafine compared with intermittent itraconazole in treatment of toenail onychomycosis. The LION Study Group. BMJ. 1999;318(7190):1031-1035.

10. Sporanox (itraconazole) [package insert], Pricara, Division of OrthoMcNeil-Janssen Pharmaceuticals, Inc., Raritan, NJ, 2011.
11. Scher RK, Breneman D, Rich P, et al. Once-weekly fluconazole (150, 300 , or $450 \mathrm{mg}$ ) in the treatment of distal subungual onychomycosis of the toenail. J Am Acad Dermatol. 1998;38(6 pt 2):S77-S86.

12. Baran R, Sigurgeirsson B, de Berker D, et al. A multicentre, randomized, controlled study of the efficacy, safety and cost-effectiveness of a combination therapy with amorolfine nail lacquer and oral terbinafine compared with oral terbinafine alone for the treatment of onychomycosis with matrix involvement. Br J Dermatol. 2007;157(1): 149-157.

13. Polak A. Preclinical data and mode of action of amorolfine. Dermatology. 1992;184(Suppl 1):3-7.

14. Belenky P, Camacho D, Collins JJ. Fungicidal drugs induce a common oxidative-damage cellular death pathway. Cell Rep. 2013;3(2): 350-358.

15. Lee RE, Liu TT, Barker KS, Lee RE, Rogers PD. Genome-wide expression profiling of the response to ciclopirox olamine in Candida albicans. J Antimicrob Chemother. 2005;55(5):655-662.

16. Penlac nail lacquer (ciclopirox) topical solution $8 \%$ [package insert], Dermik Laboratories, Berwyn, PA, 2003.

17. Jo Siu WJ, Tatsumi Y, Senda H, et al. Comparison of in vitro antifungal activities of efinaconazole and currently available antifungal agents against a variety of pathogenic fungi associated with onychomycosis. Antimicrob Agents Chemother. 2013;57(4):1610-1616.

18. Ogura H, Kobayashi H, Nagai K, et al. Synthesis and antifungal activities of (2R,3R)-2-aryl-1-azolyl-3-(substituted amino)-2-butanol derivatives as topical antifungal agents. Chem Pharm Bull. 1999;47(10): 1417-1425.

19. Rodriguez RJ, Low C, Bottema CD, Parks LW. Multiple functions for sterols in Saccharomyces cerevisiae. Biochim Biophys Acta. 1985;837(3):336-343.

20. Parks LW, Smith SJ, Crowley JH. Biochemical and physiological effects of sterol alterations in yeast - a review. Lipids. 1995;30(3):227-230.

21. Tatsumi Y, Nagashima M, Shibanushi T, et al. Mechanism of action of efinaconazole, a novel triazole antifungal agent. Antimicrob Agents Chemother. 2013;57(5):2405-2409.

22. Council \USAN. Statement on a Nonproprietary Name Adopted by the USAN Council: Efinaconazole. Available from: http://www.ama-assn. org/resources/doc/usan/efinaconazole.pdf. Accessed April 28, 2015.

23. LLC VPNA. Jublia (Efinaconazole) Package Insert. Bridgewater, NJ, USA; 2014. Available from: http://www.accessdata.fda.gov/drugsatfda_docs/label/2014/203567s000lbl.pdf.

24. Kircik LH. Enhancing transungual delivery and spreading of efinaconazole under the nail plate through a unique formulation approach. J Drugs Dermatol. 2014;13(12):1457-1461.

25. Sakamoto M, Sugimoto N, Kawabata H, et al. Transungual delivery of efinaconazole: its deposition in the nail of onychomycosis patients and in vitro fungicidal activity in human nails. J Drugs Dermatol. 2014;13(11):1388-1392.

26. Elewski BE, Pollak RA, Pillai R, Olin JT. Access of efinaconazole topical solution, $10 \%$, to the infection site by spreading through the subungual space. J Drugs Dermatol. 2014;13(11):1394-1398.

27. Arika T, Yokoo M, Hase T, Maeda T, Amemiya K, Yamaguchi H. Effects of butenafine hydrochloride, a new benzylamine derivative, on experimental dermatophytosis in guinea pigs. Antimicrob Agents Chemother. 1990;34(11):2250-2253.

28. Tatsumi Y, Yokoo M, Arika T, Yamaguchi H. In vitro antifungal activity of KP-103, a novel triazole derivative, and its therapeutic efficacy against experimental plantar tinea pedis and cutaneous candidiasis in guinea pigs. Antimicrob Agents Chemother. 2001;45(5): 1493-1499.

29. Tatsumi Y, Yokoo M, Arika T, Yamaguchi H. KP-103, a novel triazole derivative, is effective in preventing relapse and successfully treating experimental interdigital tinea pedis and tinea corporis in guinea pigs. Microbiol Immunol. 2002;46(7):425-432.

30. Tatsumi Y, Yokoo M, Arika T, Yamaguchi H. In vivo fungicidal effect of KP-103 in a guinea pig model of interdigital tinea pedis determined by using a new method for removing the antimycotic carryover effect. Microbiol Immunol. 2002;46(7):433-439. 
31. Tatsumi Y, Yokoo M, Senda H, Kakehi K. Therapeutic efficacy of topically applied KP-103 against experimental tinea unguium in guinea pigs in comparison with amorolfine and terbinafine. Antimicrob Agents Chemother. 2002;46(12):3797-3801.

32. Sugiura K, Sugimoto N, Hosaka S, et al. The low keratin affinity of efinaconazole contributes to its nail penetration and fungicidal activity in topical onychomycosis treatment. Antimicrob Agents Chemother. 2014;58(7):3837-3842.

33. Iwata $\mathrm{A}$, Watanabe $\mathrm{Y}$, Kumagai $\mathrm{N}$, et al. In vitro and in vivo assessment of dermatophyte acquired resistance to efinaconazole, a novel triazole antifungal. Antimicrob Agents Chemother. 2014;58(8):4920-4922.

34. Lipner S, Scher RK. Onychomycosis: current and future therapies. Cutis. 2014;93(2):60-63.

35. Diao Y, Zhao R, Deng X, Leng W, Peng J, Jin Q. Transcriptional profiles of Trichophyton rubrum in response to itraconazole. Med Mycol. 2009; 47(3):237-247.

36. Jarratt M, Siu WJ, Yamakawa E, Kodera N, Pillai R, Smith K. Safety and pharmacokinetics of efinaconazole $10 \%$ solution in healthy volunteers and patients with severe onychomycosis. J Drugs Dermatol. 2013; 12(9):1010-1016.

37. Hardin TC, Graybill JR, Fetchick R, Woestenborghs R, Rinaldi MG, Kuhn JG. Pharmacokinetics of itraconazole following oral administration to normal volunteers. Antimicrob Agents Chemother. 1988;32(9): 1310-1313.

38. Kovarik JM, Mueller EA, Zehender H, Denouel J, Caplain H, Millerioux L. Multiple-dose pharmacokinetics and distribution in tissue of terbinafine and metabolites. Antimicrob Agents Chemother. 1995;39(12): $2738-2741$.

39. Tschen EH, Bucko AD, Oizumi N, Kawabata H, Olin JT, Pillai R. Efinaconazole solution in the treatment of toenail onychomycosis: a phase 2, multicenter, randomized, double-blind study. $J$ Drugs Dermatol. 2013;12(2):186-192.

40. Elewski BE, Rich P, Pollak R, et al. Efinaconazole 10\% solution in the treatment of toenail onychomycosis: two phase III multicenter, randomized, double-blind studies. J Am Acad Dermatol. 2013;68(4): 600-608.

41. Del Rosso JQ, Reece B, Smith K, Miller T. Efinaconazole 10\% solution: a new topical treatment for onychomycosis: contact sensitization and skin irritation potential. J Clin Aesthet Dermatol. 2013;6(3):20-24.

42. Jo W, Glynn M, Nejishima H, et al. Nonclinical safety assessment of efinaconazole solution (10\%) for onychomycosis treatment. Regul Toxicol Pharmacol. 2014;70(1):242-253.

43. Glynn M, Jo W, Minowa K, et al. Efinaconazole: developmental and reproductive toxicity potential of a novel antifungal azole. Reprod Toxicol. 2014;52:18-25.

44. Robbins JM. Treatment of onychomycosis in the diabetic patient population. J Diabetes Complications. 2003;17(2):98-104.
45. Vlahovic TC, Joseph WS. Efinaconazole topical, $10 \%$ for the treatment of toenail onychomycosis in patients with diabetes. J Drugs Dermatol. 2014;13(10):1186-1190.

46. Jennings MB, Pollak R, Harkless LB, Kianifard F, Tavakkol A. Treatment of toenail onychomycosis with oral terbinafine plus aggressive debridement: IRON-CLAD, a large, randomized, open-label, multicenter trial. J Am Podiatr Med Assoc. 2006;96(6):465-473.

47. Lipner SR, Scher RK. Onychomycosis. In: Razzaghi-Abyaneh M, editor. Medical Mycology: Past, Present and Future. USA: CRC; In press 2015.

48. Markinson BC, Caldwell BD, Efinaconazole Topical Solution, 10\%: Efficacy in Patients with Onychomycosis and Coexisting Tinea Pedis. J Am Podiatr Med Assoc. Epub 2015 Apr 13.

49. Szepietowski JC, Reich A, Garlowska E, Kulig M, Baran E; Onychomycosis Epidemiology Study Group. Factors influencing coexistence of toenail onychomycosis with tinea pedis and other dermatomycoses: a survey of 2761 patients. Arch Dermatol. 2006; 142(10):1279-1284.

50. Rich P. Efinaconazole topical solution, $10 \%$ : the benefits of treating onychomycosis early. J Drugs Dermatol. 2015;14(1):58-62.

51. Zeichner JA, Stein Gold L, Korotzer A. Penetration of ((14)C)efinaconazole topical solution, $10 \%$, does not appear to be influenced by nail polish. J Clin Aesthet Dermatol. 2014;7(9):34-36.

52. Zeichner JA, Stein Gold L, Korotzer A. Erratum: penetration of (14C)-efinaconazole $10 \%$ solution does not appear to be influenced by nail polish. J Clin Aesthet Dermatol. 2014;7(11):8.

53. Schein JR, Gause D, Stier DM, Lubeck DP, Bates MM, Fisk R. Onychomycosis. Baseline results of an observational study. $J \mathrm{Am}$ Podiatr Med Assoc. 1997;87(11):512-519.

54. Chacon A, Franca K, Fernandez A, Nouri K. Psychosocial impact of onychomycosis: a review. Int J Dermatol. 2013;52(11):1300-1307.

55. Tosti A, Elewski BE. Treatment of onychomycosis with efinaconazole $10 \%$ topical solution and quality of life. J Clin Aesthet Dermatol. 2014;7(11):25-30.

56. Patel T, Dhillon S. Efinaconazole: first global approval. Drugs. 2013;73(17):1977-1983.

57. Ltd KPC. R\&D Pipeline; 2013 [January 25, 2015]. Available from: http://www.kaken.co.jp/english/rd_pipeline.html.

58. Inc VPI. Valeant Pharmaceuticals Announces Receipt of US FDA Complete Response Letter for Efinaconazole; 2013 [January 26, 2014]. Available from: http://ir.valeant.com/investor-relations/ news-releases/news-release-details/2013/Valeant-PharmaceuticalsAnnounces-Receipt-Of-US-FDA-Complete-Response-Letter-ForEfinaconazole.
Infection and Drug Resistance

\section{Publish your work in this journal}

Infection and Drug Resistance is an international, peer-reviewed openaccess journal that focuses on the optimal treatment of infection (bacterial, fungal and viral) and the development and institution of preventive strategies to minimize the development and spread of resistance. The journal is specifically concerned with the epidemiology of antibiotic

\section{Dovepress}

resistance and the mechanisms of resistance development and diffusion in both hospitals and the community. The manuscript management system is completely online and includes a very quick and fair peerreview system, which is all easy to use. Visit http://www.dovepress.com/ testimonials.php to read real quotes from published authors. 